MIT-CTP-2346

hep-th/9408053

August, 1994

\title{
BACKGROUND INDEPENDENT ALGEBRAIC STRUCTURES IN CLOSED STRING FIELD THEORY
}

\author{
Ashoke SeN * \\ Tata Institute of Fundamental Research \\ Homi Bhabha Road, Bombay 400005, India \\ and \\ BARTON ZWIEBACH $^{\dagger}$ \\ Center for Theoretical Physics, LNS and Department of Physics \\ MIT, Cambridge, Massachusetts 02139, U.S.A.
}

\begin{abstract}
We construct a Batalin-Vilkovisky (BV) algebra on moduli spaces of Riemann surfaces. This algebra is background independent in that it makes no reference to a state space of a conformal field theory. Conformal theories define a homomorphism of this algebra to the BV algebra of string functionals. The construction begins with a graded-commutative free associative algebra $\mathcal{C}$ built from the vector space whose elements are orientable subspaces of moduli spaces of punctured Riemann surfaces. The typical element here is a surface with several connected components. The operation $\Delta$ of sewing two punctures with a full twist is shown to be an odd, second order derivation that squares to zero. It follows that $(\mathcal{C}, \Delta)$ is a BatalinVilkovisky algebra. We introduce the odd operator $\delta=\partial+\hbar \Delta$, where $\partial$ is the boundary operator. It is seen that $\delta^{2}=0$, and that consistent closed string vertices define a cohomology class of $\delta$. This cohomology class is used to construct a Lie algebra on a quotient space of $\mathcal{C}$. This Lie algebra gives a manifestly background independent description of a subalgebra of the closed string gauge algebra.
\end{abstract}

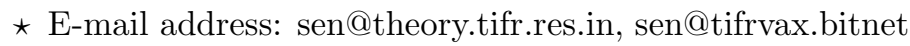

$\dagger$ E-mail address: zwiebach@irene.mit.edu, zwiebach@mitlns.bitnet.

Supported in part by D.O.E. contract DE-AC02-76ER03069. 


\section{Introduction and Summary}

At present the formulation of closed string field theory requires two choices. A choice of a set of string vertices, and a choice of a conformal field theory representing a string background. It is now known that the use of two different nearby sets of string vertices leads to the same string field theory [1]. Furthermore the use of two nearby conformal field theories also leads to the same string field theory [2]. This latter property is called background independence. Since a fundamental goal in string theory is the writing of a manifestly background independent formulation of the theory, investigation of background independent structures is an important endeavor.

In our earlier work [2] we found Riemann surfaces analogs of the antibracket and the delta operator of Batalin-Vilkovisky (BV) quantization. By making no reference to the state space of a conformal theory, such objects define a background independent structure. We also indicated that by including disconnected Riemann surfaces one would obtain a complete BV algebra structure. The definition and main properties of BV algebras have been considered in Refs. [ 3,4,5]. The relevance of the Riemann surface BV algebra is that a conformal background furnishes a natural map (homomorphism) to the BV algebra of string functionals. Therefore, the Riemann surface BV algebra is a background independent object that underlies the background dependent BV algebra of string fields.

There are three main points to the present work, and we discuss them now:

(i) We give a detailed and precise description of the relevant complex $\mathcal{C}$ of subspaces of moduli spaces of disconnected punctured Riemann surfaces, and introduce a graded associative algebra. We prove the existence of a BV algebra by introducing a delta operator $\Delta$ which is shown to be a second order derivation that squares to zero. This part of the work gives an economical derivation of the results of [2] and completes some of the details that were not given there.

(ii) It was found in [2] that the exponential of the formal sum of closed string vertices $e^{\mathcal{V} / \hbar}$ defines an element of $\mathcal{C}$ that is annihilated by the operator $\delta=$ $\partial+\hbar \Delta$, where $\partial$ is the boundary operator (picks the boundary of spaces of surfaces). Moreover, $\delta^{2}=0$. We were led to believe that the problem of finding a consistent set of string vertices can be reformulated as the problem of finding a cohomology class of $\delta$ on the complex $\mathcal{C}$. We use here the earlier work of Ref.[1] to show that given two nearby sets of consistent string vertices $\mathcal{V}$ and $\mathcal{V}^{\prime}$, the difference $e^{\mathcal{V} / \hbar}-e^{\mathcal{V}^{\prime} / \hbar}$ is indeed $\delta$ trivial. In obtaining this result we had to include in the 
complex $\mathcal{C}$ some formal limits of spaces of surfaces. This part of the work clarifies the geometrical basis of the independence of string field theory on the choice of string vertices and confirms the identification of string vertices with a cohomology class.

(iii) In Ref.[6] we discussed the Lie algebra of gauge transformations of a quantum field theory formulated in the BV approach. These gauge transformations are built using the antibracket, the delta operator and the master action. We build a background independent Lie algebra by using the geometrical antibracket, delta operator, and the string vertices $\mathcal{V}$ (which are, except for the kinetic term, the geometrical representative of the string action). The Lie algebra is shown to be independent of the representative $e^{\mathcal{V} / \hbar}$ for the cohomology class of $\delta$. We show that the natural map from spaces of surfaces to string functionals furnished by a conformal background defines a homomorphism between the two Lie algebras.

There are some obvious and fundamental questions that we will not address here. We have obtained a background independent BV algebra and Lie algebra, and a homomorphism to background dependent BV algebras and Lie algebras defined on string functionals. We would like to know by how much the homomorphism fails to be surjective. If the failure is small the background independent algebraic structures discussed here capture much of the string field theory algebraic structure. It is also not clear how to use the background independent structures to give a manifestly background independent construction of string field theory. Witten [7] has suggested that the space of homomorphisms between the Riemann surface and string field BV algebras is a plausible candidate for the space of two-dimensional field theories. This idea, and variations thereof deserve concrete examination.

This paper is organized as follows. In sect. 2 we construct the complex $\mathcal{C}$ and introduce the relevant associative algebra. In sect. 3 we construct the BV algebra on $\mathcal{C}$, discuss the $\partial$ and $\delta$ operators, review the homomorphism to the BV algebra of string functionals, and introduce the operations of contraction and Lie derivatives on $\mathcal{C}$. In sect. 4 we discuss cohomology of $\delta$ in $\mathcal{C}$, changes of string vertices, and the background independent Lie algebra built using the string vertices. 


\section{The Associative Graded-Commutative Algebra}

Let us begin by defining the spaces we are going to work with. We let $\mathcal{M}_{n}^{g}$ denote the moduli space of Riemann surfaces of genus $g$ and with $n$ punctures. The space $\mathcal{P}_{n}^{g}$ will denote the moduli space of Riemann surfaces of genus $g$ and with $n$ punctures with a chosen analytic coordinate at each puncture. The space $\mathcal{P}_{n}^{g}$ is infinite dimensional (except when $n=0$ ) as infinite number of parameters are needed to define coordinates around punctures. The space $\mathcal{P}_{n}^{g}$ has the structure of a fiber bundle over $\mathcal{M}_{n}^{g}$, with a projection that consists of forgetting about the analytic coordinates at the punctures.

In closed string field theory it is useful to introduce the space $\widehat{\mathcal{P}}_{n}^{g}$ which is also a space fibered over $\mathcal{M}_{n}^{g}$. This space is obtained from $\mathcal{P}_{n}^{g}$ by a projection that forgets the phase of the local coordinate at each puncture. These spaces are useful because they admit globally defined sections that extend all the way to the boundary of $\mathcal{M}_{n}^{g}$. This is not the case for $\mathcal{P}_{n}^{g}$. The spaces $\widehat{\mathcal{P}}_{n}^{g}$ are also infinite dimensional, except when $n=0$.

We will see that the main geometrical operation having to do with the BV antibracket is the operation of twist-sewing. This is a natural operation on $\widehat{\mathcal{P}}$ where we do not have the phases available to do sewing with a fixed sewing parameter. In fact, an antibracket in $\mathcal{P}$ defined by twist-sewing would be degenerate and thus unacceptable. Therefore we will only analyze the BV structure on $\widehat{\mathcal{P}}$. We will begin by setting up the vector space $\mathcal{C}$ where the algebra is defined, and then introduce the dot product.

\subsection{The Vector Space}

We will be interested in finite dimensional orientable subspaces of $\widehat{\mathcal{P}}_{n}^{g}$ for all $g, n \geq 0$. Those spaces will be called basic spaces, to distinguish them from generalized spaces to be introduced later. These subspaces may or may not have boundaries, and may or may not be connected, but will be taken to be smooth submanifolds. A single surface of some genus $g$ and some number of punctures $n$, is a basic space of zero dimension; a one parameter family of surfaces is a basic space of dimension one. The dimension of a basic space can easily exceed that of the moduli space $\mathcal{M}_{n}^{g}$; in such case the basic space must contain families of surfaces that give the same underlying surface upon forgetting about the local coordinates at the punctures. For $n=0$ the dimensionality of a basic space cannot exceed that of $\mathcal{M}_{0}^{g}(\equiv 6 g-6)$. 
Let us now introduce the space $\widehat{\mathcal{P}}_{\left(n_{1}, \cdots n_{r}\right)}^{\left(g_{1}, \cdots g_{r}\right)}$ which will be defined as the cartesian product of a finite number of decorated moduli spaces mentioned above. We take

$$
\widehat{\mathcal{P}}_{\left(n_{1}, \cdots n_{r}\right)}^{\left(g_{1}, \cdots g_{r}\right)} \equiv \widehat{\mathcal{P}}_{n_{1}}^{g_{1}} \times \ldots \times \widehat{\mathcal{P}}_{n_{r}}^{g_{r}}
$$

A point in this space is a collection of surfaces $\left(\Sigma_{1}, \ldots \Sigma_{r}\right)$ where $\Sigma_{i} \in \widehat{\mathcal{P}}_{n_{i}}^{g_{i}}$. We will think of $\left(\Sigma_{1}, \ldots \Sigma_{r}\right)$ as a single generalized Riemann surface, that is a surface with $r$ disconnected components. The space $\widehat{\mathcal{P}}_{\left(n_{1}, \cdots n_{r}\right)}^{\left(g_{1}, \cdots g_{r}\right)}$ can therefore be thought of as a space of disconnected Riemann surfaces. Given a collection $\mathcal{A}_{g_{i}, n_{i}} \subset \widehat{\mathcal{P}}_{g_{i}, n_{i}}$ of basic spaces of surfaces, we can easily define a subspace of disconnected surfaces. We introduce the product space

$$
\mathcal{A}_{n_{1}}^{g_{1}} \times \cdots \times \mathcal{A}_{n_{r}}^{g_{r}} \in \widehat{\mathcal{P}}_{\left(n_{1}, \cdots n_{r}\right)}^{\left(g_{1}, \cdots g_{r}\right)}
$$

This subspace of disconnected surfaces must also have its punctures labeled. Each disconnected surface has $N=\sum n_{k}$ punctures and they must be labeled from 1 to $N$. To start with we just have $r$ connected components, with the $i$-th component having its punctures labeled from 1 to $n_{i}$. Let $P_{k}^{(i)}$ denote the $k$-th puncture of the space $\mathcal{A}_{n_{i}}^{g_{i}}$. In the relabeled object this will become the puncture $P_{k+\sum_{j<i} n_{j}}$. This defines the labeling of the punctures in the disconnected surfaces. Orientation. Let $\left[\mathcal{A}_{n_{i}}^{g_{i}}\right]$ denote, at any point of $\mathcal{A}_{n_{i}}^{g_{i}}$, an ordered basis of tangent vectors to $\mathcal{A}_{n_{i}}^{g_{i}} \subset \widehat{\mathcal{P}}_{n_{i}}^{g_{i}}$. This globally defined basis of ordered vectors defines the orientation of $\mathcal{A}_{n_{i}}^{g_{i}}$. Let $\left\{\mathcal{A}_{n_{i}}^{g_{i}}\right\}$ denote the tangent vectors in $\widehat{\mathcal{P}}_{\left(n_{1}, \cdots n_{r}\right)}^{\left(g_{1}, \cdots g_{r}\right)}$ induced by the tangent vectors $\left[\mathcal{A}_{n_{i}}^{g_{i}}\right]$ of $\mathcal{A}_{g_{i}, n_{i}}$. Then the orientation of $\mathcal{A}_{n_{1}}^{g_{1}} \times \cdots \times \mathcal{A}_{n_{r}}^{g_{r}}$ is defined by the ordered set of tangent vectors $\left[\left\{\mathcal{A}_{n_{1}}^{g_{1}}\right\}, \ldots\left\{\mathcal{A}_{n_{r}}^{g_{r}}\right\}\right]$.

Symmetric Spaces. As usual with spaces of surfaces with labeled punctures there is a useful notion of a symmetric space. A basic space of surfaces $\mathcal{A}$ is said to be symmetric if the space obtained by exchanging the labels of any two labeled punctures is exactly the same as the original space. It follows from our definition of a product space that the punctures are labeled in a specific way and under the exchange of labels the space is not invariant. In order to define a notion of a symmetric product space, we now introduce a symmetrized product, where we

\footnotetext{
$\star$ By an abuse of notation we shall often refer to the puncture on a Riemann surface associated with a subspace $\mathcal{A}$ of the moduli space as a puncture of the space $\mathcal{A}$.
} 
sum over all possible ways of labeling the punctures in the resulting disconnected surfaces. We define

$$
\llbracket \mathcal{A}_{n_{1}}^{g_{1}}, \cdots, \mathcal{A}_{n_{r}}^{g_{r}} \rrbracket \equiv \frac{1}{n_{1} ! \cdots n_{r} !} \sum_{\sigma \in S_{N}} \mathbf{P}_{\sigma}\left(\mathcal{A}_{n_{1}}^{g_{1}} \times \cdots \times \mathcal{A}_{n_{r}}^{g_{r}}\right)
$$

where the sum $\sum_{\sigma \in S_{N}}$ runs over all permutations of $N$ labels, and $\mathbf{P}_{\sigma}$ denotes the operator that changes $P_{n} \rightarrow P_{\sigma(n)}$. The above sum should be understood to be a formal sum where we add up spaces multiplied by real numbers. The factors multiplying the sum in the right hand side have been introduced for later convenience. By construction, the space $\llbracket \mathcal{A}_{n_{1}}^{g_{1}}, \mathcal{A}_{n_{2}}^{g_{2}}, \cdots \mathcal{A}_{n_{r}}^{g_{r}} \rrbracket$ is left invariant under the exchange of any two labels of a pair of labeled punctures. In such a symmetric space any fixed label puncture must appear in every disconnected component of the generalized surfaces except for those components which do not carry any puncture. The orientation of the space $\llbracket \mathcal{A}_{n_{1}}^{g_{1}}, \cdots \mathcal{A}_{n_{r}}^{g_{r}} \rrbracket$ is defined by the orientation of the various terms appearing on the right hand side of Eqn.(2.3).

It follows from our definition of $\llbracket \cdots \rrbracket$ in Eqn.(2.3), that the basic space $\llbracket \mathcal{A} \rrbracket$ is symmetric even if $\mathcal{A}$ is not. Furthermore, for a symmetric basic space $\mathcal{A}$ one has $\llbracket \mathcal{A} \rrbracket=\mathcal{A}$, by virtue of the normalization factor included in (2.3). The same normalization factor guarantees that

$$
\llbracket \mathcal{A}_{n_{1}}^{g_{1}}, \cdots, \mathcal{A}_{n_{r}}^{g_{r}} \rrbracket=\llbracket \llbracket \mathcal{A}_{n_{1}}^{g_{1}} \rrbracket, \cdots, \llbracket \mathcal{A}_{n_{r}}^{g_{r}} \rrbracket \rrbracket
$$

for arbitrary basic spaces $\mathcal{A}_{n_{i}}^{g_{i}}$. Let us consider the case when all the basic spaces of surfaces appearing in Eqn.(2.3) are symmetric and have the property that any given surface $\Sigma \in \mathcal{A}_{n_{i}}^{g_{i}}$ with labeled punctures appears in $\mathcal{A}_{n_{i}}^{g_{i}}$ with unit weight. (We shall define such spaces $\mathcal{A}_{n_{i}}^{g_{i}}$ to be symmetric basic spaces with unit weight.) Then the sum in the right hand side can be rewritten as a sum over inequivalent splittings of the $N$ labels in groups of $n_{1}, n_{2}, \cdots, n_{r}$ labels. Each inequivalent splitting will appear in the sum $n_{1} ! n_{2} ! \cdots n_{r}$ ! times as identical terms by virtue of the symmetry of the basic spaces. As a consequence the sum in the right hand side can be written as a sum over inequivalent splittings, each term being a space of surfaces with unit weight. This means that each different labeled generalized surface will appear with unit weight. This simple fact will be useful to understand the consistency of the normalization factors of some of the equations we shall encounter later. 
Since we will always be considering oriented spaces (in the sense of homology) it is clear that we must have

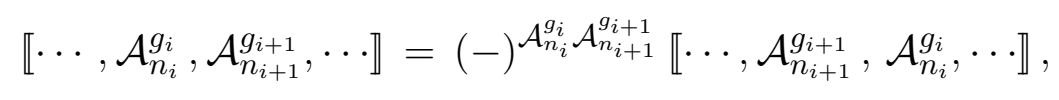

where the symbol $\mathcal{A}_{n_{i}}^{g_{i}}$ in the exponent denotes the dimension of the space $\mathcal{A}_{n_{i}}^{g_{i}}$ (this notation will be used throughout this paper). Indeed, under the exchange of the basic spaces $\mathcal{A}_{n_{i}}^{g_{i}}$ and $\mathcal{A}_{n_{i+1}}^{g_{i+1}}$, the orientation of the generalized space $\llbracket \mathcal{A}_{n_{1}}^{g_{1}}, \ldots \mathcal{A}_{n_{r}}^{g_{r}} \rrbracket$ picks up the indicated sign factor. The reader may note that the symmetric assignment of labels to the punctures is necessary for the exchange property to hold. If this was not the case the spaces to the left and to the right of (2.5) would not agree as spaces with labeled punctures.

Grading. We grade the basic spaces $\mathcal{A}_{n}^{g}$ by their dimensionality, which, as mentioned above, is a priori unrelated to the dimensionality of the moduli space $\mathcal{M}_{n}^{g}$. This $Z$ grading induces an obvious $Z_{2}$ grading according to whether the dimensionality is even or odd. The $Z$ grading of generalized subspaces is defined by the sum of the dimensionalities of the basic spaces entering the definition of the generalized space. Their $Z_{2}$ grading is also induced by the $Z$ grading.

The Complex $\mathcal{C}$. We finally introduce the complex where the BV algebra will be defined. This is the complex $\mathcal{C}$, with the structure of a vector space, whose elements, denoted as $X, Y, \cdots$ are formal sums of the form

$$
X=\sum a_{n_{1} \cdots n_{r}}^{g_{1} \cdots g_{r}} \llbracket \mathcal{A}_{n_{1}}^{g_{1}}, \cdots \mathcal{A}_{n_{r}}^{g_{r}} \rrbracket
$$

with $a_{n_{1} \cdots n_{r}}^{g_{1} \cdots g_{r}}$ a set of real numbers $(r \geq 0)$. This vector space $\mathcal{C}$ is extraordinarily large! Is is spanned by subspaces of decorated, disconnected surfaces of all genus and of all numbers of punctures. When we add subspaces of generalized Riemann surfaces simplification is only possible when the genus and number of punctures of all the basic spaces match, and, in addition, all but one of the basic spaces actually coincide. For example, the addition of $\llbracket \mathcal{A}_{n_{1}}^{g_{1}}, \mathcal{A}_{n_{2}}^{g_{2}} \rrbracket$ and $\llbracket \mathcal{B}_{n_{3}}^{g_{3}}, \mathcal{B}_{n_{4}}^{g_{4}} \rrbracket$, with all $g_{i}$ and $n_{i}$ different, cannot be simplified. Not even $\llbracket \mathcal{A}_{n_{1}}^{g_{1}}, \mathcal{A}_{n_{2}}^{g_{2}} \rrbracket+\llbracket \mathcal{B}_{n_{1}}^{g_{1}}, \mathcal{B}_{n_{2}}^{g_{2}} \rrbracket$ can be simplified in general. Nevertheless

$$
\llbracket \mathcal{A}_{n_{1}}^{g_{1}}, \mathcal{A}_{n_{2}}^{g_{2}} \rrbracket+\llbracket \mathcal{B}_{n_{1}}^{g_{1}}, \mathcal{A}_{n_{2}}^{g_{2}} \rrbracket=\llbracket \mathcal{A}_{n_{1}}^{g_{1}}+\mathcal{B}_{n_{1}}^{g_{1}}, \mathcal{A}_{n_{2}}^{g_{2}} \rrbracket .
$$

In general we simply take

$$
\llbracket \mathcal{A}_{n_{1}}^{g_{1}}, \mathcal{A}_{n_{2}}^{g_{2}}, \cdots \rrbracket+\llbracket \mathcal{B}_{n_{1}}^{g_{1}}, \mathcal{A}_{n_{2}}^{g_{2}} \cdots \rrbracket=\llbracket \mathcal{A}_{n_{1}}^{g_{1}}+\mathcal{B}_{n_{1}}^{g_{1}}, \mathcal{A}_{n_{2}}^{g_{2}}, \cdots \rrbracket
$$

where all the basic spaces implied by the dots are the same, one by one, in the three terms appearing in the equation. The zero element $\mathbf{0}$ in the vector space can 
be identified with any generalized subspace $\llbracket \mathcal{A}_{n_{1}}^{g_{1}}, \mathcal{A}_{n_{2}}^{g_{2}}, \cdots \rrbracket$, where one (or more) of the basic spaces is the empty set of surfaces.

Since the vector space $\mathcal{C}$ is spanned by symmetric elements we call $\mathcal{C}$ the space of symmetric subspaces of direct products of basic spaces. A general element in $\mathcal{C}$ is symmetric in the sense that every sector with a fixed numbers of punctures is. The complex $\mathcal{C}$ is actually spanned by symmetrized products of symmetric basic spaces. This is clear from eqn.(2.4), where an arbitrary symmetrized product is rewritten as the symmetrized product of a set of basic spaces that are symmetric. Furthermore, since each symmetric basic space can be expressed as linear combinations of symmetric basic spaces with unit weight, it follows that the complex $\mathcal{C}$ is spanned by symmetric products of symmetric basic spaces with unit weight.

\subsection{The Dot Product $(\cdot)$}

Given two vectors $X, Y \in \mathcal{C}$, whose general form was given in Eqn.(2.6), we define the dot product $X \cdot Y \in \mathcal{C}$, by the following two equations:

$$
\begin{gathered}
\llbracket \mathcal{A}_{n_{1}}^{g_{1}}, \ldots, \mathcal{A}_{n_{r}}^{g_{r}} \rrbracket \cdot \llbracket \mathcal{B}_{m_{1}}^{h_{1}}, \ldots, \mathcal{B}_{m_{s}}^{h_{s}} \rrbracket \equiv \llbracket \mathcal{A}_{n_{1}}^{g_{1}}, \ldots, \mathcal{A}_{n_{r}}^{g_{r}}, \mathcal{B}_{m_{1}}^{h_{1}}, \ldots, \mathcal{B}_{m_{s}}^{h_{s}} \rrbracket \\
\left(\sum_{i} a_{i} X_{i}\right) \cdot\left(\sum_{j} b_{j} Y_{j}\right) \equiv \sum_{i, j} a_{i} b_{j} X_{i} \cdot Y_{j}, \quad X_{i}, Y_{j} \in \mathcal{C} .
\end{gathered}
$$

It is manifest from this definition that $X \cdot Y \in \mathcal{C}$. For symmetric basic spaces $\mathcal{A}_{n_{i}}^{g_{i}}$ and $\mathcal{B}_{n_{i}}^{g_{i}}$ with unit weight, the spaces $X_{1}=\llbracket \mathcal{A}_{n_{1}}^{g_{1}}, \ldots, \mathcal{A}_{n_{r}}^{g_{r}} \rrbracket$ and $Y_{1}=\llbracket \mathcal{B}_{m_{1}}^{h_{1}}, \ldots, \mathcal{B}_{m_{s}}^{h_{s}} \rrbracket$ have the property that each inequivalent labeled surface appears with unit weight (see the remarks below Eqn.(2.3)). Since the right hand side of Eqn.(2.9) is also built as a symmetrized product of symmetric basic spaces with unit weight, it follows that in the dot product $X_{1} \cdot Y_{1}$ each inequivalent labeled surface appears with unit weight.

As defined, the dot product is manifestly associative. It also follows from the above definition, and Eqn.(2.5) that the dot product is graded commutative. In summary

$$
X \cdot Y=(-)^{X Y} Y \cdot X, \quad X \cdot(Y \cdot Z)=(X \cdot Y) \cdot Z, \quad X, Y, Z \in \mathcal{C} .
$$

We have therefore obtained the structure of a graded commutative algebra on $\mathcal{C}$. 
It is useful to have an alternative description of the dot product $(X \cdot Y)$ for the case when $X$ and $Y$ are not themselves symmetrized products but linear combinations theoreof. For this let us regard $X$ as a collection of labeled surfaces $\Sigma_{X}$ with $N$ punctures appearing with weight $w_{\Sigma_{X}}$, and similarly $Y$ as a collection of labeled surfaces $\Sigma_{Y}$ with $M$ punctures appearing with weight factor $w_{\Sigma_{Y}}$. We can then define $X \cdot Y$ as the collection of surfaces of the form

$$
w_{\Sigma_{X}} w_{\Sigma_{Y}} \cdot \frac{1}{N ! M !} \cdot \sum_{\sigma \in S_{N+M}} \mathbf{P}_{\sigma}\left(\Sigma_{X} \times \Sigma_{Y}\right) .
$$

In constructing these class of terms we have relabeled the punctures of the disjoint surfaces from one to $N+M$ and have symmetrized over all possible assignment of labels to the punctures, dividing by the symmetry factors $N$ ! and $M$ ! which take into account the original symmetry of $X$ and $Y$. This definition is extended to more general surfaces (say with variable numbers of punctures) by multilinearity. In order to show that this definition is compatible with the earlier one we must show how to derive (2.9) from (2.12). Using Eqn.(2.3) and Eqn.(2.12) we get,

$$
\begin{array}{r}
\llbracket \mathcal{A}_{n_{1}}^{g_{1}}, \cdots, \mathcal{A}_{n_{r}}^{g_{r}} \rrbracket \cdot \llbracket \mathcal{B}_{m_{1}}^{h_{1}}, \cdots, \mathcal{B}_{m_{s}}^{h_{s}} \rrbracket=\frac{1}{n_{1} ! \cdots n_{r} !} \cdot \frac{1}{m_{1} ! \cdots m_{s} !} \sum_{\sigma^{\prime} \in S_{N}} \sum_{\sigma^{\prime \prime} \in S_{M}} \sum_{\sigma \in S_{N+M}} \\
\cdot \frac{1}{N ! M !} \mathbf{P}_{\sigma} \mathbf{P}_{\sigma^{\prime}} \mathbf{P}_{\sigma^{\prime \prime}}\left(\mathcal{A}_{n_{1}}^{g_{1}} \times \cdots \times \mathcal{A}_{n_{r}}^{g_{r}} \times \mathcal{B}_{m_{1}}^{h_{1}} \times \cdots \times \mathcal{B}_{m_{s}}^{h_{s}}\right)
\end{array}
$$

where $N=\sum n_{i}$ and $M=\sum m_{i}, \sigma^{\prime}$ is a permutation of the labels 1 to $N$ of the $\mathcal{A}$ spaces, and $\sigma^{\prime \prime}$ is a permutation of the labels 1 to $M$ of the $\mathcal{B}$ spaces. Due to the sum over the $\sigma$ permutations of the labels 1 to $N+M$, the $\sigma^{\prime}$ and $\sigma^{\prime \prime}$ permutations only contribute factors of $N$ ! and $M$ ! respectively. We therefore get

$$
\begin{array}{r}
\llbracket \mathcal{A}_{n_{1}}^{g_{1}}, \cdots, \mathcal{A}_{n_{r}}^{g_{r}} \rrbracket \cdot \llbracket \mathcal{B}_{m_{1}}^{h_{1}}, \cdots, \mathcal{B}_{m_{s}}^{h_{s}} \rrbracket=\frac{1}{n_{1} ! \cdots n_{r} !} \cdot \frac{1}{m_{1} ! \cdots m_{s} !} \sum_{\sigma \in S_{N+M}} \\
\cdot \mathbf{P}_{\sigma}\left(\mathcal{A}_{n_{1}}^{g_{1}} \times \cdots \times \mathcal{A}_{n_{r}}^{g_{r}} \times \mathcal{B}_{m_{1}}^{h_{1}} \times \cdots \times \mathcal{B}_{m_{s}}^{h_{r}}\right),
\end{array}
$$

which, by Eqn.(2.3) agrees with equation (2.9). This shows that the two definitions of the dot product coincide.

It follows from Eqn.(2.12) that when we multiply two spaces $X$ and $Y$ that only contain configurations appearing with weights equal to one, the dot product $(X \cdot Y)$ is made of different configurations each appearing with unit weight (except 
in degenerate cases). This is seen as follows. Each term we consider in (2.12) would have $w_{\Sigma_{X}}=w_{\Sigma_{Y}}=1$, and we get configurations with weight factor $1 / N ! M !$. Nevertheless, by symmetry the spaces $X$ and $Y$, contain respectively $N$ ! copies of $\Sigma_{X}$ and $M$ ! copies os $\Sigma_{Y}$, with the punctures relabeled. Each of these copies can be seen to contribute an equal amount to $(X \cdot Y)$ by the rule expressed in (2.12). The number of different copies that can be combined is $N ! M !$. This cancels out the same weight factor appearing in the denominator of (2.12) and results in every single configuration produced with unit weight.

For our later developments it will be convenient, though not strictly necessary, to introduce a new element in the complex $\mathcal{C}$ which will be a unit $\mathbf{1}$ for the dot product. Thus, by definition, $X \cdot \mathbf{1}=\mathbf{1} \cdot X=X$, for every $X \in \mathcal{C}$. Intuitively, the unit can be thought to represent the surface with zero number of connected components and zero punctures. As such, under the dot product, which simply puts together the disconnected surfaces of the spaces to be multiplied, multiplication by the unit has no effect.

The reader may note that the construction of the graded-commutative associative algebra given here was done along the lines of the standard construction in mathematics of free associative algebras starting from a vector space $V$. In our case that vector space is the space of basic subspaces of Riemann surfaces. As in the standard construction one forms all tensor products $V^{\otimes N}$ and adds them together to form a complex. There is natural multiplication $V^{\otimes N} \times V^{\otimes M} \rightarrow V^{\otimes(N+M)}$. Most of the work we had to do in our construction was due to the necessity of working with symmetric spaces throughout.

\section{The Batalin Vilkovisky Algebra}

In this section we begin by defining the $\Delta$ operator and then turn to show that it squares to zero and that it is a second order derivation of the dot product. This shows that we have a Batalin-Vilkovisky algebra [3,4,5]. We discuss how the antibracket is recovered and explain the properties of the boundary operator $\partial$. We also review the homomorphism to the BV algebra of string functionals. We conclude by showing how to extend the complex $\mathcal{C}$ to include elements which are formal limits and have the interpretation of contractions and Lie derivatives. 


\subsection{THE OPERATOR $\Delta$}

While our aim in this section is to give a definition for an operator $\Delta$ acting on elements of $\mathcal{C}$ it will be convenient to introduce an operator $\Delta_{i, j}$, with $i \neq j$, that will act on direct products of basic spaces of surfaces (not necessarily symmetrized).

For any product space $\mathcal{A}$ ( a space of the form $\mathcal{A}=\mathcal{A}_{n_{1}}^{g_{1}} \times \cdots \times \mathcal{A}_{n_{r}}^{g_{r}}$ ), we define $\Delta_{i j} \mathcal{A}$ as $\frac{1}{2}$ times the set of surfaces obtained by twist sewing the punctures $P_{i}$ and $P_{j}$ of every element in $\mathcal{A}$. If $z_{i}$ and $z_{j}$ denote the local coordinates around those punctures, twist sewing means sewing through the relation $z_{i} z_{j}=e^{i \theta}$ with $0 \leq \theta \leq 2 \pi$. It is clear from the above definition that $\Delta_{i j}=\Delta_{j i}$. We extend the definition of $\Delta_{i j}$ to linear combinations of product spaces by taking it to be a linear operator. The $\Delta_{i j}$ operation reduces the number of punctures by two. Therefore in the resulting surfaces the punctures must be relabeled from 1 to $N-2$. This will be done preserving the ascending order of the punctures. If the two punctures to be sewn lie on the same connected component, that connected component increases its genus by one, and $\Delta_{i j} \mathcal{A}$ is made of disconnected surfaces with $r$ components. If the two punctures to be sewn lie on the different connected components, those two connected components fuse to give a single connected component. In this case $\Delta_{i j} \mathcal{A}$ has $r-1$ connected components. The dimension of $\Delta_{i j} \mathcal{A}$ is $\operatorname{dim}(\mathcal{A})+1$, with the twist angle $\theta$ parametrizing the extra dimension. If $\{\mathcal{A}\}$ denotes the ordered basis of tangent vectors induced on $\Delta_{i j} \mathcal{A}$ by the original basis of tangent vectors $[\mathcal{A}]$ of $\mathcal{A}$, we define the orientation of the space $\Delta_{i j} \mathcal{A}$ to be $\left[\frac{\partial}{\partial \theta},\{\mathcal{A}\}\right]^{\star}$ If $\mathcal{A}$ represents a product space with a total number of punctures less than or equal to one, $\Delta_{i j} \mathcal{A}=0$.

For any $X=\llbracket \mathcal{A}_{n_{1}}^{g_{1}}, \cdots, \mathcal{A}_{n_{r}}^{g_{r}} \rrbracket \in \mathcal{C}$, we now define

$$
\Delta X \equiv \Delta_{i j} X=\frac{1}{n_{1} ! \cdots n_{r} !} \sum_{\sigma \in S_{N}} \Delta_{i j} \mathbf{P}_{\sigma}\left(\mathcal{A}_{n_{1}}^{g_{1}} \times \cdots \times \mathcal{A}_{n_{r}}^{g_{r}}\right)
$$

where we made use of (2.3). The right hand side is actually independent of the choice of $i$ and $j(\neq i)$ in $\Delta_{i j}$. This follows from the symmetry of $X$. It is also clear that $\Delta X \in \mathcal{C}$. The linearity of $\Delta_{i j}$ implies that $\Delta$ extends to general elements of $\mathcal{C}$ as a linear operator: $\Delta \sum_{i} a_{i} X_{i} \equiv \sum_{i} a_{i} \Delta X_{i}$.

$\star$ Since the phases of the local coordinates around the punctures of $\mathcal{A}$ are not defined, the induced tangent vectors $[\mathcal{A}]$ of $\Delta_{i j} \mathcal{A}$ are defined only up to addition of terms proportional to $\partial / \partial \theta$. This ambiguity does not affect the definition of the orientation of $\Delta_{i j} \mathcal{A}$ given above. 
Let us make a comment about weight factors. If $X=\llbracket \mathcal{A}_{n_{1}}^{g_{1}}, \cdots, \mathcal{A}_{n_{r}}^{g_{r}} \rrbracket$ is made of symmetric basic spaces $\mathcal{A}_{n_{i}}^{g_{i}}$ with unit weight, as mentioned below (2.4), each different labeled surface in $X$ has unit weight. Now we claim that the same holds for $\Delta X$. This is so because whenever we pick any two punctures to be sewn in a surface in $X$, the surface with those two punctures exchanged also appears with unit weight in $X$ giving an identical contribution to $\Delta X$. The explicit one-half factor in the definition of $\Delta$ then restores unit weight.

We will define $\Delta$ to give zero on any element of $\mathcal{C}$ representing a space of disconnected surfaces with a total number of punctures less than or equal to one. Our definition of $\Delta$ does not tell us how it acts on the unit element $\mathbf{1}$ of the algebra. We will define

$$
\Delta \mathbf{1}=0
$$

and this will actually be necessary for the consistency of the BV algebra to be introduced later. It is also in accord with the intuitive notion that the surface representing $\mathbf{1}$, with no connected components and no punctures, does not admit a nontrivial action of $\Delta$.

\subsection{The BV Algebra Structure}

We shall now show that the dot product $(\cdot)$ and the $\Delta$ operator satisfy the properties defining a BV algebra.

1. The operator $\Delta$ is nilpotent:

$$
\Delta(\Delta X)=\Delta^{2} X=0
$$

Proof: This property holds, by definition for the special element $X=1$. If $X$ denotes a subspace containing three or less punctures the above property is clearly true, so we will now consider spaces whose surfaces have at least four punctures. Let $X$ be such a space and let us now calculate $\Delta^{2} X$ in two ways, first as $\Delta_{12} \Delta_{12} X$, and then as $\Delta_{12} \Delta_{34} X$. The independence of $\Delta$ from the choice of punctures guarantees that both evaluations must give the same answer. We will show that they differ by a sign, and therefore the object is identically zero.

In calculating $\Delta_{12} \Delta_{12} X$ we first twist sew punctures $P_{1}$ and $P_{2}$ of every element of $X$ and then relabel the punctures $P_{3} \cdots P_{N}$ as $P_{1} \cdots P_{N-2}$ and twist sew the new $P_{1}$ and $P_{2}$ punctures. This means that effectively the second sewing operation 
is joining the original $P_{3}$ and $P_{4}$ punctures. In calculating $\Delta_{12} \Delta_{34} X$ we first twist sew punctures $P_{3}$ and $P_{4}$ of every element of $X$, and then twist sew the punctures $P_{1}$ and $P_{2}$ which need no relabeling. Let $\Sigma_{X}$ be an arbitrary element of $X$ appearing with some fixed weight factor, and representing a surface with labeled punctures. Let $\partial / \partial \theta_{12}$ be the tangent vector associated with the sewing of the original punctures $P_{1}$ and $P_{2}$, and $\partial / \partial \theta_{34}$ be the tangent vector associated with the sewing of the original punctures $P_{3}$ and $P_{4}$. As explained above, the orientation of the space $\Delta_{12} \Delta_{12} X$ at the subspace $\Delta_{12}\left(\Delta_{12} \Sigma_{X}\right)$ will contain the tangent vectors $\left[\frac{\partial}{\partial \theta_{34}}, \frac{\partial}{\partial \theta_{12}},\{X\}\right]$ in this order. On the other hand the orientation of the space $\Delta_{12} \Delta_{34} X$ at the subspace $\Delta_{12}\left(\Delta_{34} \Sigma_{X}\right)$ will contain the tangent vectors $\left[\frac{\partial}{\partial \theta_{12}}, \frac{\partial}{\partial \theta_{34}},\{X\}\right]$ in this order. Thus the spaces $\Delta_{12}\left(\Delta_{12} \Sigma_{X}\right)$ and $\Delta_{12}\left(\Delta_{34} \Sigma_{X}\right)$ are just the same space with opposite orientation. This shows that the two ways of calculating $\Delta^{2} X$ give answers that differ by a minus sign, and hence $\Delta^{2} X=0$.

2. The operator $\Delta$ acts as a second order super-derivation on the dot product:

$$
\begin{aligned}
\Delta(X \cdot Y \cdot Z)= & \Delta(X \cdot Y) \cdot Z+(-)^{X} X \cdot \Delta(Y \cdot Z)+(-)^{(X-1) Y} Y \cdot \Delta(X \cdot Z) \\
& -\Delta X \cdot(Y \cdot Z)-(-)^{X} X \cdot(\Delta Y) \cdot Z-(-)^{X+Y} X \cdot Y \cdot \Delta Z
\end{aligned}
$$

where $X, Y$ and $Z$ are elements of $\mathcal{C}$ of definite dimensions.

Proof: By the linearity of $\Delta$ and the multilinearity of the dot product it is enough to consider the case when $X, Y$ and $Z$ are spaces of the form

$$
X=\llbracket \mathcal{X}_{l_{1}}^{f_{1}}, \cdots \mathcal{X}_{l_{r}}^{f_{r}} \rrbracket, \quad Y=\llbracket \mathcal{Y}_{m_{1}}^{g_{1}}, \cdots \mathcal{Y}_{m_{s}}^{g_{s}} \rrbracket, \quad Z=\llbracket \mathcal{Z}_{n_{1}}^{h_{1}}, \cdots \mathcal{Z}_{n_{t}}^{h_{t}} \rrbracket
$$

Moreover, since $\mathcal{C}$ is spanned by symmetrized products of symmetric basic spaces with unit weight (see discussion at the end of sec.2.1) there is no loss of generality in taking all the basic spaces appearing here to be symmetric and with unit weight. Consider now the left hand side of Eqn.(3.4):

$$
\Delta(X \cdot Y \cdot Z)=\Delta \llbracket \mathcal{X}_{l_{1}}^{f_{1}}, \cdots \mathcal{X}_{l_{r}}^{f_{r}}, \mathcal{Y}_{m_{1}}^{g_{1}}, \cdots \mathcal{Y}_{m_{s}}^{g_{s}}, \mathcal{Z}_{n_{1}}^{h_{1}}, \cdots \mathcal{Z}_{n_{t}}^{h_{t}} \rrbracket
$$

The right hand side of this equation contains a space of surfaces which breaks naturally into six distinct classes. Let us denote by $R_{X X}$ the subset of surfaces appearing in the right hand side of Eqn.(3.6) where the two punctures sewn by $\Delta$ lie both on surfaces belonging to $X$. Furthermore, let $R_{X Y}$ denote the subset of surfaces appearing in the right hand side of Eqn.(3.6) where one of the two 
punctures sewn by $\Delta$ lies on a surface belonging to $X$ and the other one on a surface belonging to $Y$. We define $R_{Y Y}, R_{Z Z}$ and $R_{Y Z}, R_{Z X}$ similarly. The orientation of the $R$ spaces is taken to be induced by the orientation of $\Delta(X \cdot Y \cdot Z)$, and therefore, is given as $\{\partial / \partial \theta,[X],[Y],[Z]\}$. This enables us to write (3.6) as

$$
\Delta(X \cdot Y \cdot Z)=R_{X X}+R_{Y Y}+R_{Z Z}+R_{X Y}+R_{Y Z}+R_{Z X} .
$$

By the property of $\Delta$ giving unit weight to inequivalent configurations when acting on a symmetrized product of symmetric basic spaces with unit weight, it follows that each inequivalent configuration appearing in the right hand side of eqn.(3.6) has unit weight. Since (3.7) is a disjoint breakup of this set, each configuration appearing in any of the $R$ classes must appear with unit weight.

We now claim that

$$
\Delta(X \cdot Y) \cdot Z=R_{X X}+R_{X Y}+R_{Y Y},
$$

As configurations it is clear that the set of surfaces on either side of the equation is contained in the set of surfaces on the other side of the equation. Moreover, the sets on the right hand side are all disjoint. The only question is whether or not for each configuration the weight factors agree. We have seen above that the right hand side contains each of its inequivalent configurations with unit weight. From our previous argument, since $(X \cdot Y)$ is a symmetrized product of symmetric basic spaces of unit weight, $\Delta(X \cdot Y)$ contains each inequivalent configuration with unit weight. Since $Z$ is also of the same type, our discussion of the dot product (below eqn. (2.14)) implies that $\Delta(X \cdot Y) \cdot Z$ must have each configuration with unit weight. Finally, the orientation of the spaces on the two sides are identical. This proves Eqn.(3.8).

In an exactly identical manner, we can derive the following two equations:

$$
\begin{gathered}
(-)^{X} X \cdot \Delta(Y \cdot Z)=R_{Y Y}+R_{Y Z}+R_{Z Z}, \\
(-)^{(X-1) Y} Y \cdot \Delta(X \cdot Z)=R_{X X}+R_{X Z}+R_{Z Z},
\end{gathered}
$$

where the extra sign factors appearing on the left hand sides of these equations are required to take into account the necessary rearrangement of the tangent vectors 
to bring them to the order $\{\partial / \partial \theta,[X],[Y],[Z]\}$. Using similar arguments we can derive three more useful equations:

$$
\begin{gathered}
\Delta X \cdot Y \cdot Z=R_{X X}, \\
(-)^{X} X \cdot \Delta Y \cdot Z=R_{Y Y}, \\
(-)^{X+Y} X \cdot Y \cdot \Delta Z=R_{Z Z} .
\end{gathered}
$$

Eqn.(3.4) follows immediately from Eqns.(3.7)-(3.13). This concludes our proof that $\Delta$ is a second order derivation of the dot product.

\subsection{Recovering the Antibracket}

It was shown in Refs. [ 8,3,4] that given a graded commutative and associative algebra with a second order derivation which squares to zero, namely, a BV algebra, one can reconstruct the standard BV antibracket. In particular, one defines the anti-bracket $\{X, Y\}$ through the relation

$$
\{X, Y\}=(-)^{X} \Delta(X \cdot Y)+(-)^{X+1}(\Delta X) \cdot Y-X \cdot(\Delta Y)
$$

then the anti-bracket satisfies the usual BV algebra relations

$$
\begin{gathered}
\{X, Y\}=-(-)^{(X+1)(Y+1)}\{Y, X\} \\
(-)^{(X+1)(Z+1)}\{\{X, Y\}, Z\}+\text { cyclic permutations of } X, Y, Z=0 .
\end{gathered}
$$

It also satisfies the following properties with respect to the dot product

$$
\{X, Y \cdot Z\}=\{X, Y\} \cdot Z+(-)^{(X+1) Y} Y \cdot\{X, Z\}
$$

Geometrical Picture. As in our analysis of subsection 3.2, we can express the contribution to $(-)^{X} \Delta(X \cdot Y)$ as sum of three different classes of surfaces. We denote by $S_{X X}$ the subset of surfaces appearing in this term where the two punctures sewn by $\Delta$ lie both on surfaces belonging to $X$. We define $S_{Y Y}$ similarly. Furthermore, let $S_{X Y}$ denote the subset of surfaces appearing in this term where one of the two 
punctures sewn by $\Delta$ lies on a surface belonging to $X$, and the other one on a surface belonging to $Y$. The orientation of the $S$ spaces is taken to be induced by the orientation of $(-)^{X} \Delta(X \cdot Y)$, and therefore, is given as $\{[X], \partial / \partial \theta,[Y]\}$. This enables us to write,

$$
(-1)^{X} \Delta(X \cdot Y)=S_{X X}+S_{Y Y}+S_{X Y}
$$

We also have, using arguments similar to the ones used in subsection 3.2,

$$
\begin{aligned}
(-)^{X}(\Delta X) \cdot Y & =S_{X X}, \\
X \cdot(\Delta Y) & =S_{Y Y} .
\end{aligned}
$$

Eqn.(3.14) now gives:

$$
\{X, Y\}=S_{X Y}
$$

Thus the antibracket $\{X, Y\}$ has the following interpretation. If $\Sigma_{X}$ denotes an element of $X$ and $\Sigma_{Y}$ denotes an element of $Y$, then $\{X, Y\}$ consists of surfaces where one puncture of $\Sigma_{X}$ is sewn to one puncture of $\Sigma_{Y}$, and the final punctured surface is symmetrized in all the external punctures. The orientation of the resulting space is given by $\{[X], \partial / \partial \theta,[Y]\}$. We therefore recover the definition of the antibracket given in Ref.[2].

From the definition (3.14) it also follows that $\{$,$\} is a bilinear operator in \mathcal{C}$

$$
\left\{\sum_{i} a_{i} X_{i}, \sum_{j} b_{j} Y_{j}\right\}=\sum_{i, j} a_{i} b_{j}\left\{X_{i}, Y_{j}\right\}, \quad X_{i}, Y_{j} \in \mathcal{C}
$$

\subsection{The Boundary Operator $\partial$}

Besides the dot product $(\cdot)$, the $\Delta$ operator, and the antibracket $\{$,$\} , there$ is another useful operator that one can define in the complex $\mathcal{C}$. For any region $\mathcal{A} \subset \widehat{\mathcal{P}}_{\left(n_{1}, \ldots, n_{r}\right)}^{\left(g_{1}, \ldots, g_{r}\right)}, \partial \mathcal{A}$ will denote the boundary of $\mathcal{A}$. The orientation of $\mathcal{A}$ induces an orientation on $\partial \mathcal{A}$ as usual. Given a point $p \in \partial \mathcal{A}$, a set of basis vectors $\left[v_{1}, \cdots v_{k}\right]$ of $T_{p}(\partial \mathcal{A})$ defines the orientation of $\partial \mathcal{A}$ if $\left[n, v_{1}, \cdots v_{k}\right]$, with $n$ a basis 
vector of $T_{p} \mathcal{A}$ pointing outwards, is the orientation of $\mathcal{A}$ at $p$. The definition of $\partial$ is extended over the whole complex $\mathcal{C}$ by treating it as a linear operator

$$
\partial\left(\sum_{i} a_{i} X_{i}\right)=\sum_{i} a_{i} \partial X_{i}, \quad X_{i} \in \mathcal{C}
$$

It is clear that acting on an element of $\mathcal{C}, \partial$ gives another element of $\mathcal{C}$. Also, $\partial$ acts as an odd derivation of the dot product

$$
\partial(X \cdot Y)=(\partial X \cdot Y)+(-)^{X}(X \cdot \partial Y)
$$

and anti-commutes with $\Delta$

$$
\Delta \partial X=-\partial \Delta X
$$

Properties (3.23) and (3.24) follow from the geometric definitions of $\partial,(\cdot)$ and $\Delta$. Finally, using Eqns.(3.14), (3.23) and (3.24) we see that $\partial$ acts as an odd derivation of the anti-bracket:

$$
\partial\{X, Y\}=\{\partial X, Y\}+(-)^{X+1}\{X, \partial Y\}
$$

Following [2] we now define an odd operator

$$
\delta \equiv \partial+\hbar \Delta
$$

and verify that it squares to zero

$$
\delta^{2}=(\partial+\hbar \Delta)^{2}=\partial^{2}+\hbar(\partial \Delta+\Delta \partial)+\hbar^{2} \Delta^{2}=0
$$

We can therefore define cohomology of $\delta$ in the complex $\mathcal{C}$. In the next section we shall see that the string vertices that define a string field theory can be naturally associated to a cohomology class of $\delta$.

\footnotetext{
$\star$ To obtain an outward vector one constructs a diffeomorphism between the neighborhood of $p$ and a suitable half-space. The outward vector is the image under the diffeomorphism of the standard normal to the half space (see, for example [9]).
} 


\subsection{Representations on the Space of Functions of String Fields}

For a string theory formulated around any specific matter conformal field theory with $c=26$, there is a natural map from the subspaces of moduli spaces of punctured Riemann surfaces to the space of functions of the string field. This map is obtained via the objects $\left\langle\Omega^{(k) g, n}\right|$, which are $\left(\widehat{\mathcal{H}}^{*}\right)^{n}$ valued $(6 g+2 n-6)+k$ forms on $\widehat{\mathcal{P}}_{g, n}$. Here $\widehat{\mathcal{H}}$ denotes the subspace of the Hilbert space of the combined matter-ghost conformal field theory, annihilated by $b_{0}^{-}$and $L_{0}^{-}$, and $\widehat{\mathcal{H}}^{*}$ is the dual Hilbert space. (For the precise definition of $\left\langle\Omega^{(k) g, n}\right|$, see Refs.[10,2].) Given an element $\llbracket \mathcal{A}_{g_{1}, n_{1}}^{\left(k_{1}\right)}, \ldots \mathcal{A}_{g_{r}, n_{r}}^{\left(k_{r}\right)} \rrbracket$ of $\mathcal{C}$, we define

$$
f\left(\llbracket \mathcal{A}_{g_{1}, n_{1}}^{\left(k_{1}\right)} \ldots \mathcal{A}_{g_{r}, n_{r}}^{\left(k_{r}\right)} \rrbracket\right)=\prod_{i=1}^{r} \frac{1}{n_{i} !} \int_{\mathcal{A}_{g_{i}, n_{i}}^{\left(k_{i}\right)}}\left\langle\Omega^{\left(k_{i}\right) g_{i}, n_{i}} \mid \Psi\right\rangle_{1} \cdots|\Psi\rangle_{n_{i}} .
$$

Here, for convenience of writing, we have not included the string field $|\Psi\rangle$ in the argument of $f$. This operation is extended to the whole complex $\mathcal{C}$ by taking

$$
f\left(\sum_{i} a_{i} X_{i}\right)=\sum_{i} a_{i} f\left(X_{i}\right)
$$

where $a_{i}$ are any set of numbers and $X_{i} \in \mathcal{C} \forall i$. The function $f(X)$ of a space $X$ of definite dimension, is grasmann even if the dimension of $X$ is even, and is grassmann odd if the dimension of $X$ is odd. The map $f$ is not defined for spaces containing zero, one, and two punctured spheres, as well as tori without punctures. For higher genus surfaces without punctures the map was given in Ref.[2]. We now claim that the standard $\Delta$ and product operations in the space of string fields are related to the corresponding operations in the moduli space in a simple manner:

$$
\begin{aligned}
f(\Delta X) & =-\Delta f(X) \\
f(X \cdot Y) & =f(X) \cdot f(Y), \quad X, Y \in \mathcal{C} .
\end{aligned}
$$

This is the homomorphism between the Riemann surface BV algebra, and the BV algebra of string functionals. It follows from (3.14) and the above equations that

$$
f(\{X, Y\})=-\{f(X), f(Y)\}
$$

The second equation of (3.30) follows immediately from the definition of $f$ in (3.28) and the definition of the dot product in (2.9). The derivation of the first equation

\footnotetext{
$\star$ The minus sign of the first equation could be eliminated if so desired, by changing the definition of either the geometrical or the functional $\Delta$ operator.
} 
is somewhat more involved. It was shown in Ref.[2] that

$$
\begin{aligned}
f(\Delta \mathcal{A}) & =-\Delta f(\mathcal{A}) \\
f(\{\mathcal{A}, \mathcal{B}\}) & =-\{f(\mathcal{A}), f(\mathcal{B})\},
\end{aligned}
$$

for symmetric basic spaces $\mathcal{A}, \mathcal{B}$. In order to establish the first equation in (3.30) an induction argument is useful. To begin with one shows that the second equations in (3.30) and (3.32), and (3.17) imply that whenever $f(\{X, \mathcal{A}\})=-\{f(X), f(\mathcal{A})\}$ holds for $\mathcal{A}$ a symmetric basic space and $X$ fixed, then $f(\{X \cdot \mathcal{B}, \mathcal{A}\})=-\{f(X$. $\mathcal{B}), f(\mathcal{A})\}$ with $\mathcal{B}$ a symmetric basic space. This fact implies that

$$
f(\{X, \mathcal{A}\})=-\{f(X), f(\mathcal{A})\}
$$

holds for arbitrary $X$ and $\mathcal{A}$ an arbitrary symmetric basic space. Using the second equation in (3.30), first equation in (3.32), (3.33) and (3.14) one can then show that whenever $f(\Delta X)=-\Delta f(X)$ holds for fixed $X$, then $f(\Delta(X \cdot \mathcal{A}))=-\Delta f(X \cdot \mathcal{A})$ holds with $\mathcal{A}$ a symmetric basic space. This fact, used in a simple induction argument, implies the first Eqn. in (3.30). This concludes our verification of the homomorphism.

\subsection{Contractions and Lie Derivatives of Spaces of Surfaces}

Given a vector field $\widehat{U}$ in $\widehat{\mathcal{P}}_{\left(n_{1}, \cdots n_{r}\right)}^{\left(g_{1}, \cdots g_{r}\right)}$, we define an operation that increases the degree of a space of surfaces by one. Given a surface $\Sigma$ we let $f_{* \widehat{U}}^{t} \Sigma$ denote the surface obtained by following the integral curve of the vector field $\widehat{U}$ a parameter length $t$. If $X$ is a subspace of $\widehat{\mathcal{P}}_{\left(n_{1}, \cdots n_{r}\right)}^{\left(g_{1}, \cdots g_{r}\right)}$ which is symmetric in all the punctures, we define

$$
I_{\widehat{U}}^{u} X \equiv\left\{f_{* \widehat{U}}^{t} X, t \in[0, u]\right\}
$$

that is, the space of surfaces obtained by taking every element of $X$ and including in the resulting set all the surfaces obtained while following the integral curves of $\widehat{U}$ a parameter length $u$. The orientation of $I_{\widehat{U}}^{u} X$ will be defined by $[\widehat{U},[X]]$. Note that this definition does not require that the vector field be defined over all of $\widehat{\mathcal{P}}_{\left(n_{1}, \cdots n_{r}\right)}^{\left(g_{1}, \cdots g_{r}\right)}$, Given a space $X$ the vector field only needs to be defined in a suitable 
neighborhood of $X$. We also define

$$
L_{\widehat{U}}^{u} X \equiv f_{* \widehat{U}}^{u} X-X,
$$

which computes the difference between the space of surfaces we get by following the integral curve a parameter distance $u$ and the original space of surfaces. It follows from the definitions given above that

$$
\partial I_{\overparen{U}}^{u} X=-I_{\widehat{U}}^{u} \partial X+L_{\overparen{U}}^{u} X,
$$

since the boundary operator picks two types of contributions, one from the boundary of $X$ (with a minus sign because both $\partial$ and $I$ are odd), and the other from the endpoints of the displacement along the integral curves of $\widehat{U}$.

We now extend our complex $\mathcal{C}$ by including in it the following formal limits

$$
\begin{aligned}
i_{\widehat{U}} X & \equiv \lim _{u \rightarrow 0} \frac{1}{u} I_{\widehat{U}}^{u} X, \\
\mathcal{L}_{\widehat{U}} X & \equiv \lim _{u \rightarrow 0} \frac{1}{u} L_{\widehat{U}}^{u} X .
\end{aligned}
$$

This defines the linear operators $i_{\widehat{U}}$ and $\mathcal{L}_{\widehat{U}}$ in the complex $\mathcal{C}$. We can now use the definitions given in eqns.(4.6) and (4.8) of Ref.[1] to verify that

$$
\begin{aligned}
\int_{\widehat{U} X} \Omega & =\int_{X} i_{\widehat{U}} \Omega, \\
\int_{\mathcal{L}_{\widehat{U}} X} \Omega & =\int_{X} \mathcal{L}_{\widehat{U}} \Omega,
\end{aligned}
$$

where, in the right hand sides $i_{\widehat{U}} \Omega$ and $\mathcal{L}_{\widehat{U}} \Omega$ denote respectively the contraction operation and Lie derivative on the canonical forms $\Omega$ appearing in Eqn.(3.28). We now impose the following identification on the new elements $i_{\widehat{U}} X$ and $\mathcal{L}_{\widehat{U}} X$ of $\mathcal{C}$ :

$$
\begin{aligned}
i_{\widehat{U}_{1}+\widehat{U}_{2}} X & =i_{\widehat{U}_{1}} X+i_{\widehat{U}_{2}} X, \\
\mathcal{L}_{\widehat{U}_{1}+\widehat{U}_{2}} X & =\mathcal{L}_{\widehat{U}_{1}} X+\mathcal{L}_{\widehat{U}_{2}} X .
\end{aligned}
$$

These identifications are compatible with Eqn.(3.38) since the contraction of forms $i_{\widehat{U}}$ and the Lie derivative of forms $\mathcal{L}_{\widehat{U}}$ are both linear on the vector field argument 
$\widehat{U}^{\star}$. In terms of the new objects Eqn.(3.36) implies that

$$
\partial i_{\widehat{U}}^{u} X=-i_{\widehat{U}}^{u} \partial X+\mathcal{L}_{\widehat{U}} X
$$

\section{Closed String Vertices as Cohomology in $\mathcal{C}$ and a Lie Algebra}

In the present section we will begin by showing that the element $e^{\mathcal{V} / \hbar} \in \mathcal{C}$, where $\mathcal{V}$ is the sum of the closed string field theory vertices, is annihilated by the odd operator $\delta$ introduced in sect.3.3. Since $\delta$ squares to zero, $e^{\mathcal{V} / \hbar}$ is a candidate for a cohomology class. We then reconsider the work of Ref.[1] and show that the difference between $e^{\mathcal{V} / \hbar}$ and $e^{\mathcal{V}^{\prime} / \hbar}$, where $\mathcal{V}$ and $\mathcal{V}^{\prime}$ are consistent string vertices, is a $\delta$-trivial term. We conclude by discussing a background independent Lie algebra that is constructed using the cohomology class $\mathcal{V}$ and is isomorphic to a subalgebra of the string field theory gauge algebra.

\subsection{Closed String Vertices as a Cohomology Class}

The vertices of a string field theory can be associated with $(6 g+2 n-6)$ dimentional subspaces $\mathcal{V}_{g, n}$ of $\widehat{\mathcal{P}}_{g, n}$, satisfying the recursion relations[10]:

$$
\partial \mathcal{V}_{g, n}=-\frac{1}{2} \sum_{\substack{g_{1}+g_{2}=g \\ n_{1}+n_{2}=n+2}}\left\{\mathcal{V}_{g_{1}, n_{1}}, \mathcal{V}_{g_{2}, n_{2}}\right\}-\Delta \mathcal{V}_{g-1, n+2}
$$

Let us now define

$$
\mathcal{V} \equiv \sum_{g, n} \hbar^{g} \mathcal{V}_{g, n} \quad \text { with } \quad\left\{\begin{array}{l}
n \geq 3 \text { for } g=0 \\
n \geq 1 \text { for } g=1 \\
n \geq 0 \text { for } g \geq 2
\end{array}\right.
$$

It then follows from (4.1) that the recursion relations can be written as

$$
\partial \mathcal{V}+\hbar \Delta \mathcal{V}+\frac{1}{2}\{\mathcal{V}, \mathcal{V}\}=0
$$

We shall now show that a $\mathcal{V}$ satisfying this equation defines a cohomology element of $\delta$. We define the exponential function of an even element $X \in \mathcal{C}$ by the

* This means that $\left(i_{\widehat{U}_{1}+\widehat{U}_{2}} X-i_{\widehat{U}_{1}} X-i_{\widehat{U}_{2}} X\right)$ is in the kernel of the homomorphism $f$ defined in eq.(3.28). Since ultimately we are interested in applying this formalism to string theory, we do not lose anything by defining this difference to be zero in $\mathcal{C}$ itself. 
usual power series

$$
\exp (X) \equiv \mathbf{1}+X+\frac{1}{2} X \cdot X+\frac{1}{3 !} X \cdot X \cdot X+\cdots
$$

It follows from Eqn.(3.23) that

$$
\partial[\exp (X)]=\partial X \cdot \exp (X)
$$

Moreover, using Eqn.(3.14) we find

$$
\Delta \exp (X)=\left(\Delta X+\frac{1}{2}\{X, X\}\right) \exp (X)
$$

From the last two equations we get,

$$
\delta \exp (X)=(\partial+\hbar \Delta) \exp (X)=\left(\partial X+\hbar \Delta X+\frac{1}{2} \hbar\{X, X\}\right) \exp (X)
$$

Making use of Eqn.(4.7) we see that we can now write the recursion relations (4.3) in the simple form

$$
\delta \exp (\mathcal{V} / \hbar)=0
$$

Thus $\exp (\mathcal{V} / \hbar)$ defines a cohomology element of $\delta$. It is clear that $\exp (\mathcal{V} / \hbar)$ is not $\delta$ trivial since the expansion begins with $\mathbf{1}$, and a term of the form $\delta Y \equiv \partial Y+\hbar \Delta Y$ can never contain a term proportional to 1 . This result, however, is not very interesting, since even for $\mathcal{V}=0, \exp (\mathcal{V} / \hbar)=\mathbf{1}$ will define a non-trivial element of the cohomology. A more interesting fact is that even $\exp (\mathcal{V} / \hbar)-1$, which is $\delta$ closed by virtue of Eqn.(4.8), is not $\delta$ trivial. Triviality would require that

$$
\hbar^{-1} \mathcal{V}+\frac{1}{2} \hbar^{-2} \mathcal{V} \cdot \mathcal{V}+\cdots=(\partial+\hbar \Delta)\{X\}
$$

More explicitly, this equation begins as

$$
\hbar^{-1} \mathcal{V}_{0,3}+\cdots=(\partial+\hbar \Delta)\{X\}
$$

If we are to obtain the zero-dimensional space $\mathcal{V}_{0,3}$ from the right hand side it cannot be from $\Delta$ since $\Delta$ always adds one dimension. Thus it must be from $\partial$. But $\mathcal{V}_{0,3}$ cannot be written as $\partial X$ for any $X$. This shows that $(\exp (\mathcal{V} / \hbar)-1)$ is not trivial. This is the precise statement we have in mind when we state that the string vertices define a cohomology class of $\delta$. 
We also note that given a set of closed string vertices satisfying the recursion relations (4.1), we can introduce a nilpotent operator $\delta_{\mathcal{V}}$ through the relation

$$
\delta_{\mathcal{V}}=\delta+\{\mathcal{V},\}
$$

which has the property

$$
\delta_{\mathcal{V}}\{\mathcal{A}, \mathcal{B}\}=\left\{\delta_{\mathcal{V}} \mathcal{A}, \mathcal{B}\right\}+(-)^{\mathcal{A}+1}\left\{\mathcal{A}, \delta_{\mathcal{V}} \mathcal{B}\right\}
$$

and,

$$
\delta\left(\mathcal{A} e^{\mathcal{V} / \hbar}\right)=(\delta \mathcal{V} \mathcal{A}) e^{\mathcal{V} / \hbar}
$$

for arbitrary subspaces $\mathcal{A}, \mathcal{B}$. This $\delta_{\mathcal{V}}$ operator will be useful later on.

\subsection{Changing the Closed String Vertices}

In string field theory, the vertices $\mathcal{V}_{g, n}$ defining the $\delta$ cohomology class $e^{\mathcal{V} / \hbar}$ are not unique. The simplest choice for the vertices appears to be that determined by the minimal area problem [10] and a simple example of a family of consistent closed string vertices is given by the simple deformation of attaching stubs to the vertices. In Ref.[1] the general situation when we have a parametrized family of consistent string vertices $\mathcal{V}_{g, n}(u)$ was studied. It was shown that for infinitesimally close string vertices, the resulting string field theories are related by an infinitesimal (though nonlinear) string field redefinition. This redefinition respects the antibracket and its explicit form was found.

With the insight that we have obtained into the string vertices, it is natural to expect that $e^{\mathcal{V}(u) / \hbar}$ actually represents the same cohomology class of $\delta$ for all values of $u$. If so, we should be able to establish a relation of the form

$$
\frac{d}{d u} e^{\mathcal{V}(u)}=\delta(\chi(u))
$$

for some $\chi$. We shall now show that this is indeed the case. 
The fact that we have a family of string vertices $\mathcal{V}(u)$ implies that the recursion relations are satisfied for each value of the parameter $u$

$$
\partial \mathcal{V}(u)=-\frac{1}{2}\{\mathcal{V}(u), \mathcal{V}(u)\}-\hbar \Delta \mathcal{V}(u)
$$

A geometrical fact established in [1] was the existence, for each moduli space, of a vector field $\widehat{U}$ such that

$$
f_{* \widehat{U}}^{u_{0}} \mathcal{V}(u)=\mathcal{V}\left(u+u_{0}\right)
$$

This vector $\widehat{U}$ was constructed recursively*

Consider now infinitesimal variations $d u$ and define

$$
\mathcal{W}(u) \equiv\left\{\mathcal{V}\left(u^{\prime}\right): u^{\prime} \in[u, u+d u]\right\}=I_{\widehat{U}}^{d u} \mathcal{V}(u)=d u i_{\widehat{U}}^{d u} \mathcal{V}(u)
$$

where, by definition, the orientation of a space $\{\mathcal{A}(t): t \in \mathcal{D}\}$ is given by the ordering $\{\partial / \partial t,[\mathcal{A}(t)]\}$ of the tangent vectors. Using Eqn.(3.36), we find

$$
\partial \mathcal{W}=\mathcal{V}(u+d u)-\mathcal{V}(u)-I_{\widehat{U}}^{d u} \partial \mathcal{V}(u)
$$

where explicitly

$$
\begin{aligned}
I_{\widehat{U}}^{d u} \partial \mathcal{V}(u) & =\left\{\partial \mathcal{V}\left(u^{\prime}\right): u^{\prime} \in[u, u+d u]\right\} \\
& =\left\{-\frac{1}{2}\left\{\mathcal{V}\left(u^{\prime}\right), \mathcal{V}\left(u^{\prime}\right)\right\}-\hbar \Delta \mathcal{V}\left(u^{\prime}\right): u^{\prime} \in[u, u+d u]\right\} \\
& =\left\{-\frac{1}{2}\left\{\mathcal{V}\left(u^{\prime}\right), \mathcal{V}\left(u^{\prime}\right)\right\}: u^{\prime} \in[u, u+d u]\right\}+\hbar \Delta \mathcal{W}(u)
\end{aligned}
$$

Consider now the region $R_{n_{1}, n_{2}}^{g_{1}, g_{2}}(u, v) \in \widehat{\mathcal{P}}_{n_{1}+n_{2}-2}^{g_{1}+g_{2}}$, corresponding to the collection of surfaces $\left\{\mathcal{V}_{g_{1}, n_{1}}(u), \mathcal{V}_{g_{2}, n_{2}}(v)\right\}$ obtained twist-sewing string vertices for fixed

* In Ref.[1] the vector $\widehat{U}$ satisfied the further requirement that the deformation of each $U(1)$ fiber in $\partial \mathcal{V}$ was defined by the deformation of the constituent surface(s) appearing in the right hand side of (4.15) and representing the basepoint of the fiber. This additional requirement is not necessary for the present proof. In Ref.[ 1] this extra requirement implied that integrals that had to be equal were so by the manifest equality of their integrands. 
nearby values of $u$ and $v^{\dagger}$ Let us now introduce two vector fields $U_{1}(u, v)$ and $U_{2}(u, v)$ on $R_{n_{1}, n_{2}}^{g_{1}, g_{2}}(u, v)$ as follows.

$$
\begin{aligned}
& f_{* U_{1}(u, v)}^{t}\left\{\mathcal{V}_{g_{1}, n_{1}}(u), \mathcal{V}_{g_{2}, n_{2}}(v)\right\}=\left\{\mathcal{V}_{g_{1}, n_{1}}(u+t), \mathcal{V}_{g_{2}, n_{2}}(v)\right\}+\mathcal{O}\left(t^{2}\right) \\
& f_{* U_{2}(u, v)}^{t}\left\{\mathcal{V}_{g_{1}, n_{1}}(u), \mathcal{V}_{g_{2}, n_{2}}(v)\right\}=\left\{\mathcal{V}_{g_{1}, n_{1}}(u), \mathcal{V}_{g_{2}, n_{2}}(v+t)\right\}+\mathcal{O}\left(t^{2}\right)
\end{aligned}
$$

These equations do not determine the vector fields $U_{1}(u, v)$ and $U_{2}(u, v)$ uniquely. One way to fix a choice is to demand that

$$
f_{* U_{1}(u, v)}^{t}\left\{\Sigma_{1} \in \mathcal{V}_{g_{1}, n_{1}}(u), \Sigma_{2} \in \mathcal{V}_{g_{2}, n_{2}}(v)\right\}=\left\{f_{* U}^{t} \widehat{\Sigma}_{1}, \Sigma_{2}\right\}+\mathcal{O}\left(t^{2}\right)
$$

and similarly for $U_{2}(u, v)$, where $\widehat{U}$ is the vector field appearing in (4.16). This defines the map of $U(1)$ classes arising from twist sewing. A map of the surfaces themselves is obtained by fixing arbitrarily the phases around the punctures to be sewn, as discussed in sect.4.7 of Ref.[1].

We will now single out two special vector fields

$$
\widehat{U}_{1} \equiv U_{1}(u, u), \quad \widehat{U}_{2} \equiv U_{2}(u, u)
$$

defined on $R_{n_{1}, n_{2}}^{g_{1}, g_{2}}(u, u)$, and we extend them arbitrarily but smoothly over some neighborhood of $R_{n_{1}, n_{2}}^{g_{1}, g_{2}}(u, u)$. We will still denote by $\widehat{U}_{1}$ and $\widehat{U}_{2}$ the extended vector fields. It follows from Eqns. (4.20) and (4.21), together with Eqns.(3.34) and (3.37) that

$$
\begin{aligned}
I_{\widehat{U}_{1}}^{d u}\{\mathcal{V}(u), \mathcal{V}(u)\} \equiv d u i_{\widehat{U}_{1}}\{\mathcal{V}(u), \mathcal{V}(u)\} & =\left\{\left\{\mathcal{V}\left(u^{\prime}\right), \mathcal{V}(u)\right\}: u^{\prime} \in[u, u+d u]\right\} \\
& =\{\mathcal{W}(u), \mathcal{V}(u)\}, \\
I_{\widehat{U}_{2}}^{d u}\{\mathcal{V}(u), \mathcal{V}(u)\} \equiv d u i_{\widehat{U}_{2}}\{\mathcal{V}(u), \mathcal{V}(u)\} & =\left\{\left\{\mathcal{V}(u), \mathcal{V}\left(u^{\prime}\right)\right\}: u^{\prime} \in[u, u+d u]\right\} \\
& =-\{\mathcal{V}(u), \mathcal{W}(u)\},
\end{aligned}
$$

The minus sign on the right hand side of the last equation can be traced to different locations of the tangent vector $\partial / \partial u$ on the two sides of the equation.

$\dagger$ Since $R_{n_{1}, n_{2}}^{g_{1}, g_{2}}(u, u)$ is assumed to be a submanifold of $\widehat{\mathcal{P}}_{n_{1}+n_{2}-2}^{g_{1}+g_{2}}, R_{n_{1}, n_{2}}^{g_{1}, g_{2}}(u, v)$ will also be a submanifold of $\widehat{\mathcal{P}}_{n_{1}+n_{2}-2}^{g_{1}+g_{2}}$ for $v$ sufficiently close to $u$. It is not clear, however, that a disjoint union of the various $R_{n_{1}, n_{2}}^{g_{1}, g_{2}}(u, v)$, for different values of $u$ and $v$ form a submanifold of $\widehat{\mathcal{P}}_{n_{1}+n_{2}-2}^{g_{1}+g_{2}}$. 
We now consider the deformation of $\{\mathcal{V}(u), \mathcal{V}(u)\}$ by the vector $\widehat{U}_{1}+\widehat{U}_{2}$. The quantity $f_{*\left(\widehat{U}_{1}+\widehat{U}_{2}\right)}^{t}\{\mathcal{V}(u), \mathcal{V}(u)\}$ corresponds to following the integral curve of the vector field $\widehat{U}_{1}+\widehat{U}_{2}$ for a parameter distance $t$. Since the vector fields $\widehat{U}_{i}$ have been defined in a neighborhood of $R(u, u)=\cup R_{n_{1}, n_{2}}^{g_{1}, g_{2}}(u, u)$, this is a well defined operation for small enough $t$. Moreover, for sufficiently small $t, f_{*\left(\widehat{U}_{1}+\widehat{U}_{2}\right)}^{t}\{\mathcal{V}(u), \mathcal{V}(u)\}$ can be obtained by first following the integral curve of the vector field $\widehat{U}_{1}$ over a distance $t$, and then, starting at that deformed surface, following the integral curve of the vector field $\widehat{U}_{2}$ over a distance $t$. This is correct to order $\mathcal{O}\left(t^{2}\right)$ since the vector fields $\widehat{U}_{1}$ and $\widehat{U}_{2}$ are smooth. We therefore have

$$
\begin{aligned}
f_{*\left(\widehat{U}_{1}+\widehat{U}_{2}\right)}^{t}\{\mathcal{V}(u), \mathcal{V}(u)\} & =f_{* \widehat{U}_{2}}^{t}\left(f_{* \widehat{U}_{1}}^{t}\{\mathcal{V}(u), \mathcal{V}(u)\}\right)+\mathcal{O}\left(t^{2}\right) \\
& =f_{* \widehat{U}_{2}}^{t}\{\mathcal{V}(u+t), \mathcal{V}(u)\}+\mathcal{O}\left(t^{2}\right)
\end{aligned}
$$

The $\widehat{U}_{2}$ in the second line refers to the smooth extension of the vector $U_{2}(u, u)$. This differs from the vector $U_{2}(u+t, u)$ by a term of order $t$. Since we are ignoring the order $t^{2}$ terms in our analysis, we can replace the $\widehat{U}_{2}$ in the above equation by $U_{2}(u+t, t)$, and then, by virtue of Eqn.(4.21), the right hand side of Eqn.(4.26) can be replaced by $\{\mathcal{V}(u+t), \mathcal{V}(u+t)\}$. This gives,

$$
f_{*\left(\widehat{U}_{1}+\widehat{U}_{2}\right)}^{t}\{\mathcal{V}(u), \mathcal{V}(u)\}=\{\mathcal{V}(u+t), \mathcal{V}(u+t)\}+\mathcal{O}\left(t^{2}\right)
$$

From Eqns.(3.34), (3.37), and (4.27) it now follows that

$\left\{\left\{\mathcal{V}\left(u^{\prime}\right), \mathcal{V}\left(u^{\prime}\right)\right\}: u^{\prime} \in[u, u+d u]\right\}=I_{\widehat{U}_{1}+\widehat{U}_{2}}^{d u}\{\mathcal{V}(u), \mathcal{V}(u)\}=d u i_{\widehat{U}_{1}+\widehat{U}_{2}}\{\mathcal{V}(u), \mathcal{V}(u)\}$

and therefore

$$
\begin{aligned}
\left\{\left\{\mathcal{V}\left(u^{\prime}\right), \mathcal{V}\left(u^{\prime}\right)\right\}: u^{\prime} \in[u, u+d u]\right\} & =d u\left(i_{\widehat{U}_{1}}+i_{\widehat{U}_{2}}\right)\{\mathcal{V}(u), \mathcal{V}(u)\} \\
& =2\{\mathcal{W}(u), \mathcal{V}(u)\}
\end{aligned}
$$

where use was made of (3.39), (4.24) and (4.25). Back to (4.19) we have

$$
I_{\widehat{U}}^{d u} \partial \mathcal{V}(u)=\{\mathcal{V}(u), \mathcal{W}(u)\}+\hbar \Delta \mathcal{W}(u)
$$


and using (4.18) and (4.11) we get

$$
\begin{aligned}
\mathcal{V}(u+d u)-\mathcal{V}(u) & =\partial \mathcal{W}+\{\mathcal{V}(u), \mathcal{W}(u)\}+\hbar \Delta \mathcal{W}(u) \\
& =\delta \mathcal{V} \mathcal{W}(u)
\end{aligned}
$$

Using Eqn.(4.13), the above equation can be rewritten as,

$$
\exp (\mathcal{V}(u+d u) / \hbar)-\exp (\mathcal{V}(u) / \hbar)=\frac{1}{\hbar} \delta(\mathcal{W} \exp (\mathcal{V} / \hbar))
$$

This shows that $\exp (\mathcal{V}(u+d u) / \hbar)$ and $\exp (\mathcal{V}(u) / \hbar)$ belong to the same cohomology class of $\delta$.

\subsection{Gauge Transformations}

Here we wish to note the existence of a background independent Lie algebra intimately connected to gauge transformations. It is not quite the usual gauge transformations, which can only be written in a background dependent way, but it is isomorphic to a subalgebra of the full gauge algebra, and could be closely related to the underlying gauge symmetry of a manifestly background independent string field theory.

In ref.[6], the space of gauge parameters was identified with the space of hamiltonian fuctions $\Lambda$ of the string field, with the identification $\Lambda \equiv \Lambda+\Delta_{S} F$. Here $\Delta_{S}=\Delta+\frac{1}{\hbar}\{S, \cdot\}$ is the delta operator associated to the measure $d \mu \exp (2 S / \hbar)$ [11]. We then had a Lie algebra $\mathcal{L}_{G T}$ of gauge transformations defined by a bracket $[\cdot, \cdot]$

$$
\begin{aligned}
{\left[\Lambda_{1}, \Lambda_{2}\right] } & \equiv\left\{\Lambda_{1}, \Delta_{S} \Lambda_{2}\right\} \\
& =(-)^{\Lambda_{1}}\left\{\Delta_{S} \Lambda_{1}, \Lambda_{2}\right\} \\
& =\frac{1}{2}\left(\left\{\Lambda_{1}, \Delta_{S} \Lambda_{2}\right\}-(-)^{\Lambda_{1} \Lambda_{2}}\left\{\Lambda_{2}, \Delta_{S} \Lambda_{1}\right\}\right)
\end{aligned}
$$

Note that since $\Delta_{S}$ acts as an odd derivation of the anti-bracket [ 6], the differences between the various right hand sides of the above equation are all $\Delta_{S}$ exact, and hence vanish in the space of gauge parameters. Furthermore, if we add a $\Delta_{S}$ exact quantity to either $\Lambda_{1}$ or $\Lambda_{2}$, then by virtue of the nilpotence of $\Delta_{S},\left[\Lambda_{1}, \Lambda_{2}\right]$ defined in the above equation changes by a $\Delta_{S}$ exact quantity. 
The homomorphism between the Riemann surface BV algebra and the string field BV algebra is easily shown to imply that [2] for any $X \in \mathcal{C}$

$$
f\left(\delta_{\mathcal{V}} X\right)=-\Delta_{S} f(X),
$$

where $\delta_{\mathcal{V}}=\partial+\hbar \Delta+\{\mathcal{V}, \cdot\}$. This suggests a way to obtain a Lie algebra at the level of Riemann surfaces by a construction similar to that given above. At the level of Riemann surfaces we now form the space $\mathcal{C}_{\mathcal{V}}$ of equivalence classes $X \approx X+\delta_{\mathcal{V}} Y$ in $\mathcal{C}$ and in this space define a Lie algebra $\mathcal{L}_{R S}$

$$
\begin{aligned}
{\left[X_{1}, X_{2}\right] } & \equiv\left\{X_{1}, \delta_{\mathcal{V}} X_{2}\right\} \\
& =(-)^{X_{1}}\left\{\delta_{\mathcal{V}} X_{1}, X_{2}\right\} \\
& =\frac{1}{2}\left(\left\{X_{1}, \delta_{\mathcal{V}} X_{2}\right\}-(-)^{X_{1} X_{2}}\left\{X_{2}, \delta_{\mathcal{V}} X_{1}\right\}\right)
\end{aligned}
$$

As before, the difference between various lines of the above equation vanishes in $\mathcal{C}_{\mathcal{V}}$, and, furthermore, $\left[X_{1}, X_{2}\right]$ depends only on the representative classes of $X_{1}$ and $X_{2}$ in $\mathcal{C}_{\mathcal{V}}$. The Jacobi identity

$$
(-)^{X_{1} X_{3}}\left[\left[X_{1}, X_{2}\right], X_{3}\right]+\text { cyclic permutations of } X_{1}, X_{2}, X_{3}=0,
$$

can be verified using the following three equations:

$$
\begin{aligned}
& (-)^{X_{1} X_{3}}\left[\left[X_{1}, X_{2}\right], X_{3}\right]=(-)^{X_{3}}(-)^{\left(X_{1}+1\right) X_{3}}\left\{\left\{X_{1}, \delta \mathcal{V} X_{2}\right\}, \delta \mathcal{V} X_{3}\right\} \\
& (-)^{X_{2} X_{1}}\left[\left[X_{2}, X_{3}\right], X_{1}\right]=(-)^{X_{3}}(-)^{\left(X_{1}+1\right) X_{2}}\left\{\left\{\delta_{\mathcal{V}} X_{2}, \delta \mathcal{V} X_{3}\right\}, X_{1}\right\} \\
& (-)^{X_{2} X_{3}}\left[\left[X_{3}, X_{1}\right], X_{2}\right]=(-)^{X_{3}}(-)^{X_{2} X_{3}}\left\{\left\{\delta_{\mathcal{V}} X_{3}, X_{1}\right\}, \delta_{\mathcal{V}} X_{2}\right\}
\end{aligned}
$$

and the Jacobi identity (3.16) for the BV anti-bracket.

By virtue of (4.34) $f$ induces a well defined map between the Riemann surface Lie algebra $\mathcal{L}_{R S}$ and the Lie algebra of gauge transformations $\mathcal{L}_{G T}$. The map is actually a homomorphism. Indeed

$$
\begin{aligned}
{\left[f\left(X_{1}\right), f\left(X_{2}\right)\right] } & =\left\{f\left(X_{1}\right), \Delta_{S} f\left(X_{2}\right)\right\}, \\
& =-\left\{f\left(X_{1}\right), f\left(\delta_{\mathcal{V}} X_{2}\right)\right\}, \\
& =f\left(\left\{X_{1}, \delta_{\mathcal{V}} X_{2}\right\}\right) \\
& =f\left(\left[X_{1}, X_{2}\right]\right)
\end{aligned}
$$

where use was made of Eqns.(4.34) and (3.31). This homomorphism is clearly not an isomorphism. The hamiltonians of the form $\left\langle\omega_{12}|\Lambda| \Psi\right\rangle$, that generate the 
usual gauge transformations with parameter $|\Lambda\rangle$, are missing. This is so because the map $f$ is not defined for one-punctured spheres and we therefore cannot get hamiltonians linear on the string field. While linear hamiltonians can be obtained from higher genus surfaces with one puncture, it seems clear they are not general enough to reproduce all possible standard gauge transformations. In particular, since all one point functions conserve momentum (and other quantum numbers) one cannot get a $|\Lambda\rangle$ with non-zero momentum. We therefore expect that $f\left(\mathcal{L}_{R S}\right)$ is only a subalgebra $\widehat{\mathcal{L}}_{G T}$ of $\mathcal{L}_{G T}$. By the standard property of homomorphisms $\widehat{\mathcal{L}}_{G T}$ is isomorphic to the quotient Lie algebra $\mathcal{L}_{R S} / \operatorname{Ker}(f)$, where $\operatorname{Ker}(f)$ is the ideal of $\mathcal{L}_{R S}$ generated by the elements that map to zero.

Now, as has been emphasized before, the choice of $\mathcal{V}$ is not unique, but there are whole families of vertices that satisfy the recursion relations (4.1) and hence can be used to construct a closed string field theory. We shall now argue that the Lie algebra of gauge transformations defined above is independent of the choice of $\mathcal{V}$. Consider another lie algebra $\mathcal{L}^{\prime}$ defined on the complex $\mathcal{C}_{\mathcal{V}^{\prime}}$ of equivalence classes $X \approx X+\delta_{\mathcal{V}^{\prime}} Y$, where $\mathcal{V}^{\prime}=\mathcal{V}+\delta_{\mathcal{V}} \mathcal{W}$. In this algebra

$$
\left[X_{1}, X_{2}\right]^{\prime}=\left\{X_{1}, \delta \mathcal{V}^{\prime} X_{2}\right\}
$$

where

$$
\delta_{\mathcal{V}^{\prime}}=\delta \mathcal{V}+\{\delta \mathcal{V} \mathcal{W}, \cdot\}
$$

Consider the map $m: \mathcal{C} \rightarrow \mathcal{C}$ defined as

$$
m(X)=X+\{X, \mathcal{W}\}
$$

where we consider $\mathcal{W}$ to be small. This map gives an automorphism of the dot algebra

$$
m(X \cdot Y)=m(X) \cdot m(Y)
$$

as one verifies using (3.17). It is not, however, an automorphism of the BV algebra. 
Moreover using the Jacobi identity of the antibracket one sees that

$$
\{m(X), m(Y)\}=m(\{X, Y\}) .
$$

We now verify that

$$
\begin{aligned}
\delta_{\mathcal{V}^{\prime}} m(X) & =\delta_{\mathcal{V}} m(X)+\left\{\delta_{\mathcal{V}} \mathcal{W}, m(X)\right\} \\
& =\delta_{\mathcal{V}} X+\delta_{\mathcal{V}}\{X, \mathcal{W}\}+\left\{\delta_{\mathcal{V}} \mathcal{W}, X\right\} \\
& =\delta_{\mathcal{V}} X+\left\{\delta_{\mathcal{V}} X, \mathcal{W}\right\}
\end{aligned}
$$

and therefore

$$
\delta_{\mathcal{V}^{\prime}} m(X)=m\left(\delta_{\mathcal{V}} X\right)
$$

This means that the map $m$ induces a map from $\mathcal{C}_{\mathcal{V}}$ to $\mathcal{C}_{\mathcal{V}^{\prime}}$, since zero elements are mapped to zero elements. Since the map $m$ is invertible, the isomorphism between $\mathcal{L}$ and $\mathcal{L}^{\prime}$ is established if we show that

$$
\left[m\left(X_{1}\right), m\left(X_{2}\right)\right]^{\prime}=m\left(\left[X_{1}, X_{2}\right]\right) .
$$

This is now quite simple:

$$
\begin{aligned}
{\left[m\left(X_{1}\right), m\left(X_{2}\right)\right]^{\prime} } & =\left\{m\left(X_{1}\right), \delta_{\mathcal{V}^{\prime}} m\left(X_{2}\right)\right\} \\
& =\left\{m\left(X_{1}\right), m\left(\delta_{\mathcal{V}} X_{2}\right)\right\} \\
& =m\left(\left\{X_{1}, \delta_{\mathcal{V}} X_{2}\right\}\right) \\
& =m\left(\left[X_{1}, X_{2}\right]\right)
\end{aligned}
$$

And this concludes the proof that the Lie algebra $\mathcal{L}_{R S}$ is universal in the sense that it does not depend on the specific choice of consistent string vertices.

Acknowledgements: We would like to thank S. Mukhi and E. Witten for useful discussions. A. Sen would like to thank the hospitality of Center for Theoretical Physics, M.I.T., where part of this work was done. B. Zwiebach would like to thank the hospitality of the Aspen Center where this work was completed. 


\section{REFERENCES}

1. H. Hata and B. Zwiebach, 'Developing the covariant Batalin-Vilkovisky approach to string theory', Ann. Phys. 229 (1994) 177, hep-th/9301097.

2. A. Sen and B. Zwiebach, 'Quantum background independence of closed string field theory', MIT preorint CTP\#2244, hep-th/9311009; to appear in Nucl. Phys. B

3. E. Getzler, 'Batalin-Vilkovisky algebras and two-dimensional topological field theories', MIT Math preprint, hep-th/9212043.

4. M. Penkava and A. Schwarz, "On Some Algebraic Structures Arising in String Theory", UC Davis preprint, UCD-92-03, hep-th/9212072.

5. B. H. Lian and G. Zuckerman, "New Perspectives on the BRST-algebraic structure of string theory", hep-th/9211072, Yale preprint, November 1992.

6. A. Sen and B. Zwiebach, 'A note on gauge transformations in BatalinVilkovisky theory', Phys. Lett. B320 (1994) 29, hep-th/9309027.

7. E. Witten, private communication, unpublished.

8. E. Witten, 'A note on the antibracket formalism', Mod. Phys. Lett. A5 (1990) 487.

9. M. Spivak, "Calculus on Manifolds", Benjamin/Cummings publishing (1965).

10. B. Zwiebach, 'Closed string field fheory: Quantum action and the BatalinVilkovisky master equation', Nucl. Phys B390 (1993) 33, hep-th/9206084.

11. A. Schwarz, 'Geometry of Batalin-Vilkovisky quantization', UC Davis preprint, hep-th/9205088, July 1992. 